\title{
TV/Series
}

14 | 2018

Posthumains en séries

\section{Dollhouse de Joss Whedon (FOX, 2009-2010) : Écho, un " corps-marchandise " posthumain au service de la sérialité audiovisuelle}

\section{Julien Achemchame}

\section{(2) OpenEdition Journals \\ Édition électronique \\ URL : http://journals.openedition.org/tvseries/3139 \\ DOI : 10.4000/tvseries.3139 \\ ISSN : 2266-0909 \\ Éditeur \\ GRIC - Groupe de recherche Identités et Cultures}

Référence électronique

Julien Achemchame, "Dollhouse de Joss Whedon (FOX, 2009-2010) : Écho, un « corps-marchandise » posthumain au service de la sérialité audiovisuelle », TV/Series [En ligne], 14 | 2018, mis en ligne le 31 décembre 2018, consulté le 22 avril 2019. URL : http://journals.openedition.org/tvseries/3139; DOI : 10.4000/tvseries.3139

Ce document a été généré automatiquement le 22 avril 2019

\section{(c) (i) (9)}

TV/Series est mis à disposition selon les termes de la licence Creative Commons Attribution - Pas d'Utilisation Commerciale - Pas de Modification 4.0 International. 


\title{
Dollhouse de Joss Whedon (FOX, 2009-2010) : Écho, un « corps- marchandise " posthumain au service de la sérialité audiovisuelle
}

\author{
Julien Achemchame
}

1 Créée par Joss Whedon en 2009, Dollhouse est une série de science-fiction diffusée durant deux saisons sur le réseau FOX. Dollhouse est le nom d'une entreprise clandestine qui, grâce à une technologie futuriste, transforme temporairement (généralement pour une durée contractuelle prédéfinie de cinq années) des individus "volontaires", à qui l'on promet une importante somme d'argent et une félicité retrouvée après la fin du contrat, en "poupées humaines", véritables êtres posthumains. En modifiant leur architecture neuronale et en effaçant leurs personnalités originelles, l'entreprise transforme ainsi les individus en "systèmes d'exploitation" vivants capables de recevoir à l'infini de nouvelles personnalités (au préalable stockées sur des disques durs). Ces individus, appelés «Dolls» à l'état de repos (« Poupées » dans la version française) ou «Actives » («Réactifs») lorsqu'on leur a implanté une personnalité pour une mission particulière, sont ainsi réduits à l'état d'esclave au service des désirs et fantasmes en tous genres d'une riche clientèle. Au sein de cette maison de poupée, nous suivons le personnage d'Écho, autrefois Caroline Farrell, une jeune femme ayant une prédisposition génétique singulière qui la rend résistante aux multiples effacements mémoriels, appelés « traitements », subis à la suite des missions qu'elle effectue. Écho (interprétée par Eliza Dushku) gardera ainsi la mémoire de son périple au sein de la maison Dollhouse et du récit Dollhouse ainsi que des multiples personnalités qu'on lui aura implantées. Cette mémoire impossible à effacer permet ainsi la (re)construction de son identité : l'intrigue principale repose donc sur l'émancipation progressive d'Écho.

2 Série feuilletonnante, décrite par Joss Whedon comme un croisement entre Alias ${ }^{1}$ et Code Quantum ${ }^{2}$, Dollhouse propose ainsi une sérialité hybride avec des micro-récits autonomes hebdomadaires (les missions de l'active Écho) ainsi qu'une intrigue au long cours, 
complexe, et prenant pour centre l'entreprise Dollhouse, dont nous allons apprendre, comme dans toute (bonne) série d'espionnage, qu'elle n'est que l'infime partie d'un plus large "complot», fomenté à l'échelle mondiale par une société nommée Rossum Corporation, sur le point de créer une société posthumaine apocalyptique.

Dès lors, nous verrons comment la série s'appuie sur cette posthumanité technologique particulière qui permet de transformer l'humain en machine, en séparant son esprit de son corps et en détruisant son identité propre. Nous verrons d'abord de quelle posthumanité traite la série, son origine et les implications sociales globales qui en découlent, notamment à travers la figure du personnage d'Écho, en quête d'émancipation de sa servitude machinique. Nous aborderons ensuite la dimension réflexive à l'œuvre dans la série, via la posthumanité, et qui permet de voir symboliquement que derrière la Dollhouse et son système, se cache l'industrie hollywoodienne. Nous verrons en quoi cette description critique d'Hollywood est paradoxalement au service de l'actrice principale Eliza Dushku, la série lui servant de « véhicule ». Nous verrons également comment cette réflexivité travaille la matière même de la sérialité narrative télévisuelle ou, pour le dire autrement, comment le posthumain permet la réflexion sur les possibilités narratives propres à la sérialité audiovisuelle, la technologie scientifique d'implantation de personnalités permettant une potentialité narrative de reformulation de la série à l'infini, et créant ainsi une sorte de " postsérie ».

\section{Quelle est la société posthumaine de Dollhouse?}

4 S'appuyant sur les travaux de chercheurs en cybernétique et en intelligence artificielle tels que Marvin Minsky, Ray Kurzweil ou Hans Moravec, la série Dollhouse construit son univers fictionnel sur l'idée centrale d'une capacité technologique de téléchargement de la conscience humaine vers un ordinateur ou un disque dur ${ }^{3}$. Comme l'explique Dominique Lecourt :

Selon les techno-prophètes [cités précédemment], il serait possible d'expliquer avec toute la précision scientifique voulue l'intégralité des fonctions cérébrales par les modèles mathématiques qu'élaborent les spécialistes de l'intelligence artificielle. Ils affirment que les «réseaux neuronaux » peuvent être considérés comme équivalant au « hardware », la pensée constituant le « software ${ }^{4}$ ».

Ces termes de «hardware» (« ordinateur ») et «software » (« logiciel ») sont d'ailleurs ceux exacts utilisés par le personnage central du neuroscientifique et informaticien Topher Brink (Fran Kranz) pour expliquer son travail dans l'épisode «A Love Supreme » (S02E08) (cf. figure 1). 


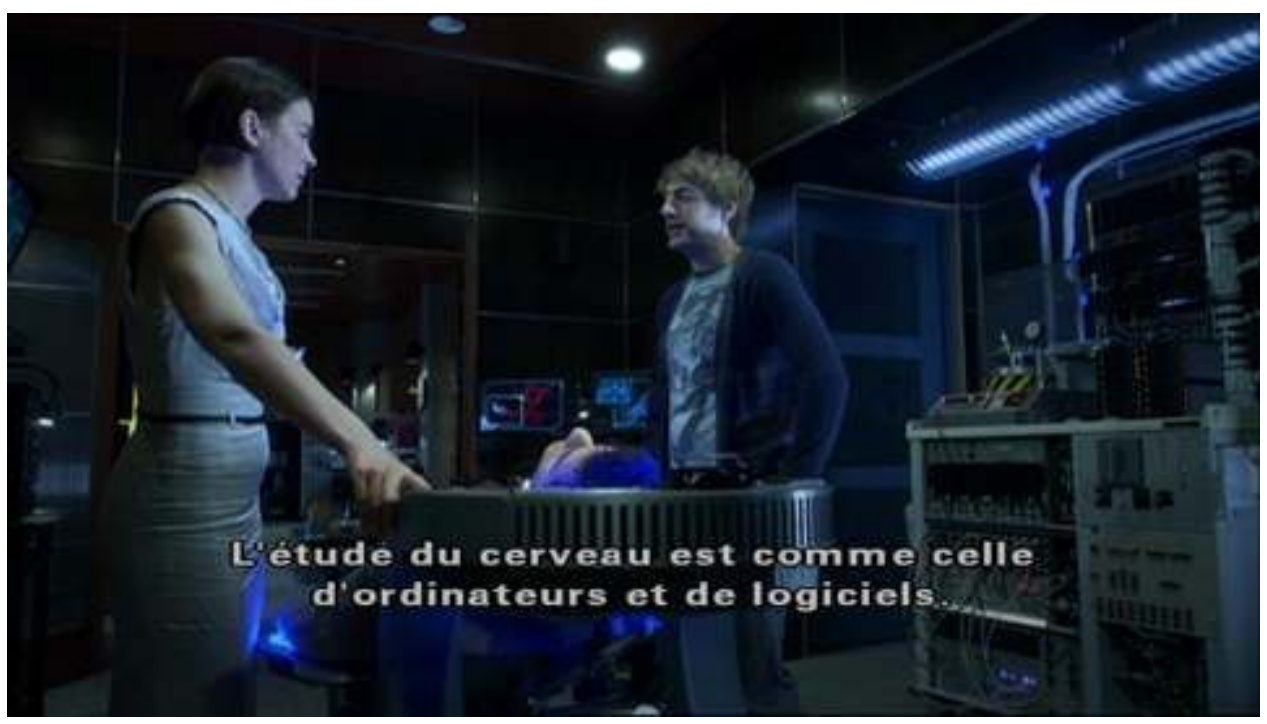

Fig. 1 : I'humain e(s)t la machine

L'originalité de la série de Joss Whedon est d'y ajouter que les personnalités téléchargées puissent être ensuite envoyées vers des hôtes humains dont le cerveau a été lui-même préalablement transformé en un ordinateur. Cette transformation de l'humain en machine, ou en " poupée » selon la terminologie utilisée dans la série, est l'aboutissement inévitable d'une numérisation de l'esprit humain: si l'on peut télécharger informatiquement une personnalité humaine, c'est donc que le cerveau humain est, à la base, à un (super)ordinateur que l'on peut programmer. Pour Topher Brink, comme il l'explique à Écho dans l'épisode "Needs " (S01E08), «le cerveau est une carte mère naturelle. Toutes nos pensées, nos sentiments et actions sont des impulsions électriques. Je ne fais que pirater le système. » L'humain est donc réifié, comparé à un ordinateur dont on pourrait prendre le contrôle pour en programmer les fonctionnalités. Les " poupées » de Dollhouse, transformées alors en « Actives » reçoivent, avec leurs « implantations » de personnalités, des capacités nouvelles ou améliorées (d'intelligence, de combat, d'endurance) mais aussi, paradoxalement, des « défauts » qui les rendent plus humaines (par exemple asthme et myopie lorsqu'Écho devient Eleanor Penn, une négociatrice en kidnapping, dans le pilote « Ghost »).

Dans l'esprit des «techno-prophètes " évoqués précédemment, le parallèle entre le cerveau et l'ordinateur, permettant le téléchargement d'une personnalité sur un disque dur, va de pair avec l'idée de dématérialisation et de virtualité de l'esprit en ce qu'il n'est donc plus nécessaire d'utiliser le corps humain, ce dernier étant irrémédiablement périssable. Dès lors l'individu demeure immortel, arpentant un "réseau neuronal informatique virtuel». Au contraire, dans Dollhouse, la "poupée » Écho est certes un récipient vide, un système d'exploitation en attente de logiciels pour fonctionner, mais c'est aussi et avant tout un corps humain dont on prend le plus grand soin. Dans un cadre agréable aux allures orientales et zen, les corps des "poupées" sont régulièrement auscultés et soignés, notamment après les missions. On leur sert de la nourriture de qualité. Elles prennent régulièrement des douches chaudes, vont au sauna, font de l'exercice, se font masser, se relaxent à l'aide de tai chi ou de yoga, taillent des bonzaïs ou peignent. Leurs activités mettent en lumière la recherche constante d'un bien-être autant mental que physique qui donne (ironiquement?) une image séduisante de ces esclaves 
posthumains, dont les corps sont clairement des marchandises de luxe dont il faut prendre soin (cf. figures $2 \mathrm{a}, 2 \mathrm{~b}, 2 \mathrm{c}$ ).
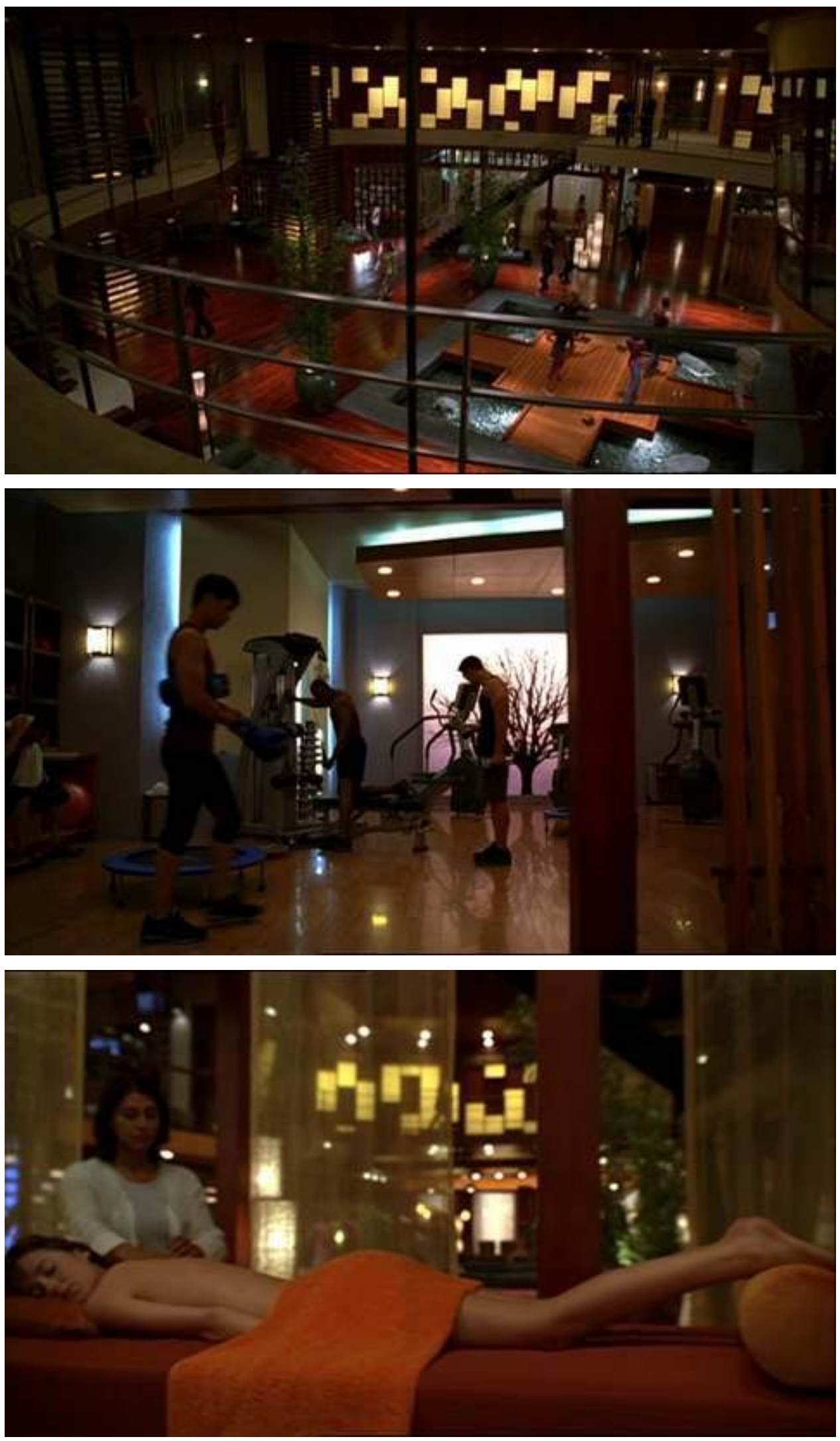

Fig. 2a, 2b, 2c : Le « corps-marchandise » soigné des « poupées » (S01E01) 


\section{Quel est le statut posthumain d'Écho?}

8 Écho est bien un être posthumain puisqu'elle est, comme le définit François-Ronan Dubois, « un être humain dont le corps est affecté par un ou plusieurs processus de modifications techno-scientifiques 5 .» Toutefois, elle n'est ni un cyborg, ni un androïde, ni un clone. Elle n'est ni le fruit d'une manipulation génétique ni celui d'une fusion entre corps humain et machine. Elle ne présente ni modification visible de son corps, ni prothèse externe. Seul son cerveau a été modifié ${ }^{6}$, la transformant en machine informatique, mais la laissant finalement terriblement humaine. (L'absence d'altération physique du corps du personnage, j'y reviendrai plus loin, l'inscrit d'autant plus fortement dans notre réalité de téléspectateurs).

Écho pourrait se définir comme du Bétail, selon la terminologie de Thierry Hoquet :

Bétail désigne [...] un type d'être artificiel mobilisant uniquement des composants organiques. Robot et Bétail interrogent toujours, en arrière plan, la question de l'individualité. [...]. Bétail désigne [...] une forme d'outil organique artificiel: il porte la marque des finalités humaines [...]. Une histoire mettant en scène Bétail décrit la trajectoire d'un être qui aspire à être individué [...]. Tout Bétail est un individu à qui l'on dénie son statut autonome et qu'on a produit pour qu'il serve une certaine fin, mais qui fait soudain montre d'une autonomie qui surprend son créateur. Par extension, la fiction de Bétail inclut le Golem et le Monstre de Frankenstein. Elle met en scène la manière dont Bétail prend conscience de sa condition, puis se rebelle contre elle et tente à toutes forces d'y échapper [...]. [...]. Ce qui fait l'intérêt commun des fictions de Robots ou de Bétail, c'est que des moyens prétendent soudain se poser comme fins; ils nous montrent la conquête de l'autonomie et la révolte contre l'aliénation?

10 Écho, être posthumain aliéné à l'ère de l'ultra-libéralisme, est bien ainsi réduite à un statut de «corps-marchandise » qui se soumet aux fantasmes d'une caste sociale aisée. Les «poupées » sont des esclaves modernes, "volontaires », et interrogent tout à la fois notre utilisation de la technologie mais aussi plus généralement le fonctionnement de notre monde contemporain, comme nous pouvons le voir dans la première séquence de l'épisode «Man on the Street » (S01E06), écrit et réalisé par Joss Whedon, où, sous la forme d'un reportage journalistique, agrémenté d'interviews, de multiples personnages évoquent la possibilité d'une Dollhouse à Los Angeles. Si une jeune femme noire, filmée en plan rapproché épaule, dénonce de manière virulente l'esclavagisme rampant qui se cache derrière l'entreprise, une autre, blanche, filmée en plan américain et arborant sur sa poitrine un badge au nom de Jessica, semble, elle, intéressée à l'idée de pouvoir échanger sa liberté contre de l'argent et une certaine jouissance facile (cf. figures 3a, 3b). C'est toute l'ambiguïté morale du propos développé par la série autour de la servitude qui est synthétisée ici : le dispositif réflexif choisi (le reportage journalistique avec microtrottoir) s'adresse directement au téléspectateur, obligé de s'interroger sur son rapport moral à la servitude. Peut-on librement choisir de devenir esclave (ou de se soumettre) ? N'est-on finalement pas déjà esclave à des degrés divers sans le savoir? 

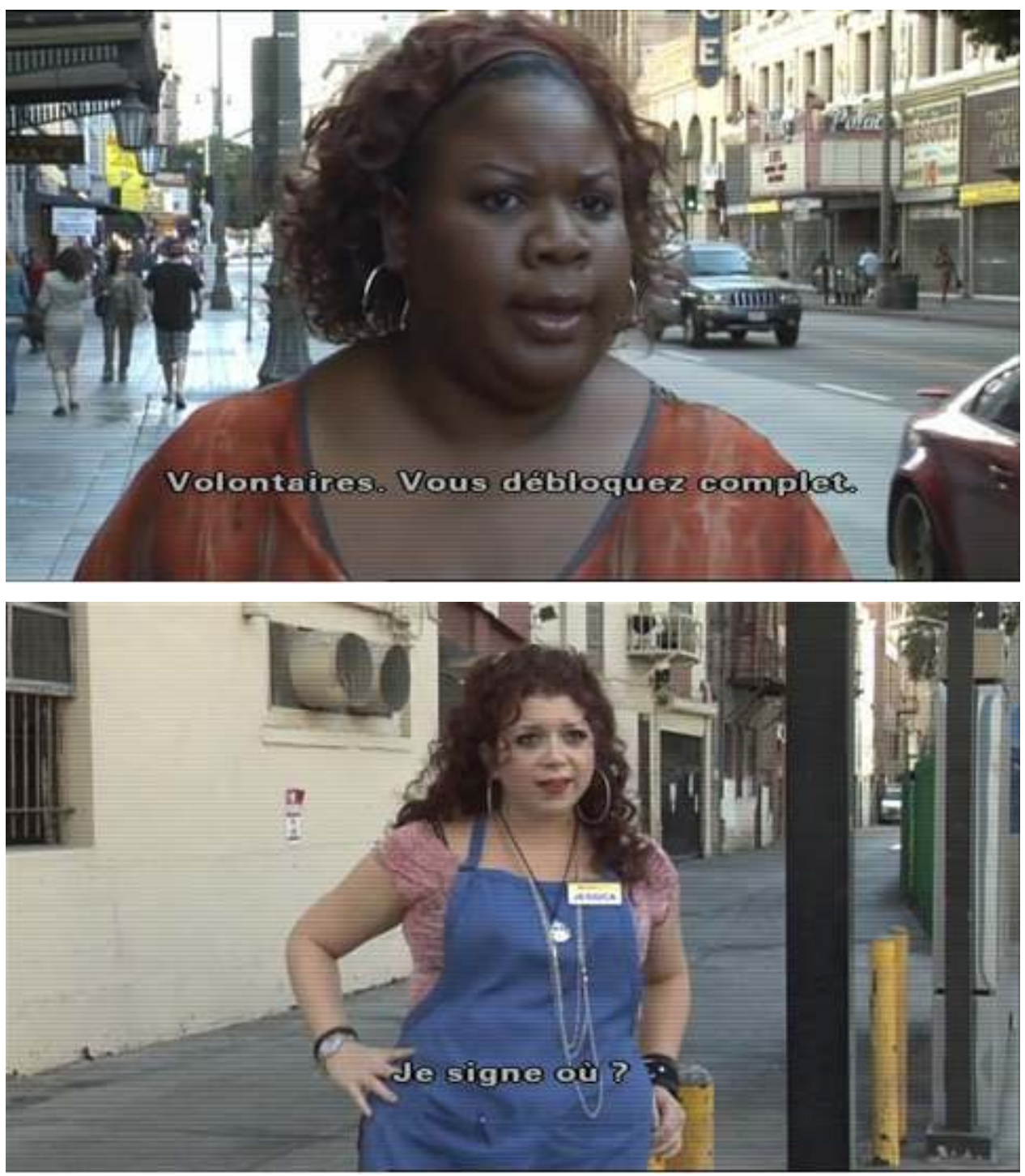

Fig. 3a, 3b : Dispositif réflexif et ambiguïté morale

11 Écho incarne admirablement (dans tous les sens du terme: physiquement et symboliquement) le « virus post-humain » qu'évoque Ophélie Hernandez :

Le «virus post-humain» est indétectable, invisible, partout à la fois. Sa stratégie ne prend plus la forme d'une incarnation monstrueuse [...]. Désincarné, le virus que nous avons engendré en engendrant une société de surconsommation effrénée s'incarne dans chacun de nos corps et s'infiltre insidieusement dans nos cerveaux. [...] [C]e sont [...] la publicité, les productions de l'industrie audiovisuelle, les médias qui insufflent à coup de messages paradoxaux, générateurs de clivage, un idéal du moi collectif. Les images publicitaires invitent à la fois à ne rien se refuser tout en se responsabilisant. Elles prônent un double discours : l'absence de frustration, la désinhibition totale, la consommation à l'excès et paradoxalement un mode de vie sain, la perfection physique et mentale, apparaissant incompatibles avec la première injonction ${ }^{8}$.

12 Cet idéal impossible secrété par notre société contemporaine est incarné par Écho dont la mémoire sans cesse effacée permet (temporairement et illusoirement) la réconciliation des deux injonctions hédoniste (statut d'Active) et stoïciste (statut de Doll). Écho est au 
carrefour de toutes les ambiguïtés: comme le dit un personnage de psychanalyste téléchargé dans la poupée Victor (Enver Gjokaj) dans l'épisode "A Love Supreme" (S02E08), elle est à la fois « putain et vierge » (voir figure 4). Écho est un personnage qui, grâce à sa posthumanité, demeure profondément paradoxal et dévoile les contradictions de notre société. Elle est finalement bien plus complexe que son apparence lisse et sa vacuité ontologique ne le laissent apparaître.

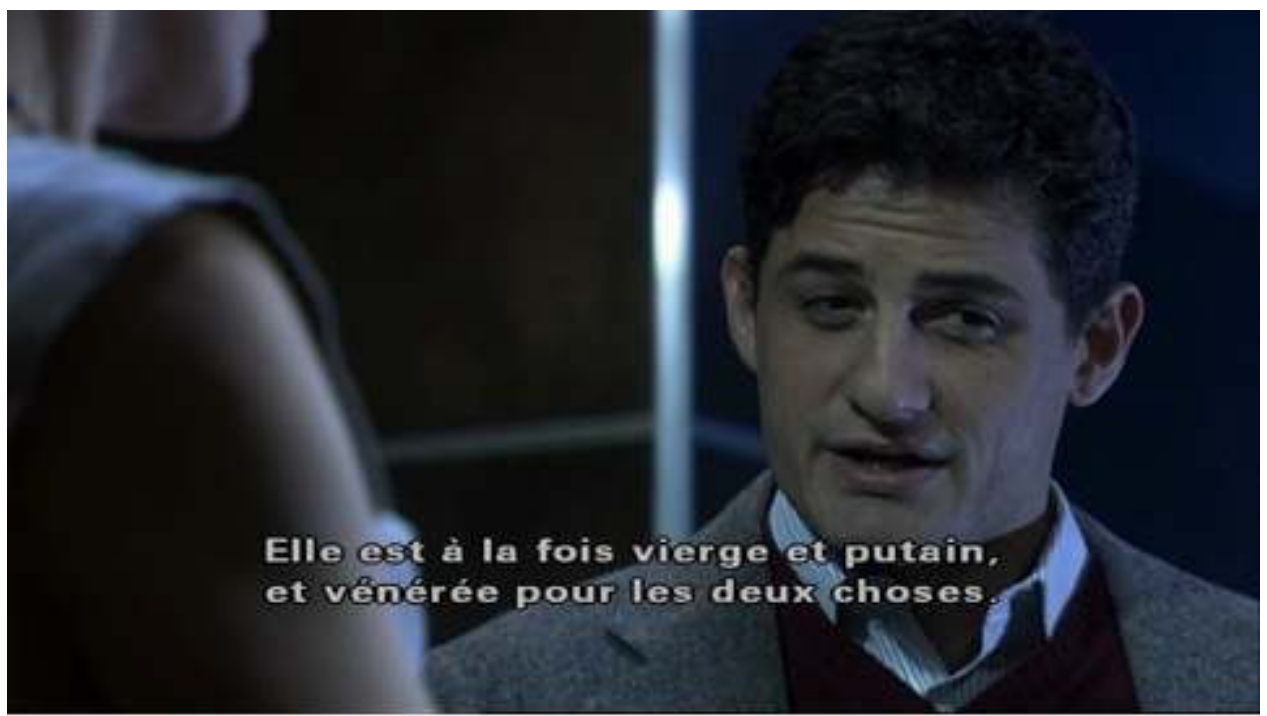

Fig. 4 : Écho, au carrefour de toutes les ambiguiités (S02E08)

\section{La trajectoire du personnage d'Écho : de l'asservissement à l'émancipation}

13 D'ailleurs, Écho, dans Dollhouse, prend son nom à la fois de la fable mythologique mais aussi, plus prosaïquement, du langage militaire. Elle est ainsi la résurgence d'un personnage féminin condamné à la dépossession de soi, à la scission entre sa voix et son corps : Écho, victime d'Héra (ou de Pan selon la version du mythe), est invisible, caisse de résonance vide des sons qui lui sont envoyés. Dans la série, elle est paradoxalement un corps visible mais creux, dont les voix multiples changent au gré des implantations de personnalités qui la contrôlent successivement. Elle est également un soldat docile capable d'accomplir les missions les plus difficiles.

14 Écho est en effet au service d'un conglomérat pharmaceutique appelé Rossum dont le nom vient explicitement, de l'aveu même de son créateur Clyde Randolph, dans l'épisode "Getting Closer » (S02E11), de R.U.R. (Rossum's Universal Robots, la pièce de science-fiction du dramaturge tchèque Karel Čapek écrite en 1921) : «Rossum is just a name, actually. From a play. Although technically you're not robots, it seemed to fit. » (cf. figures 5a, 5b). 

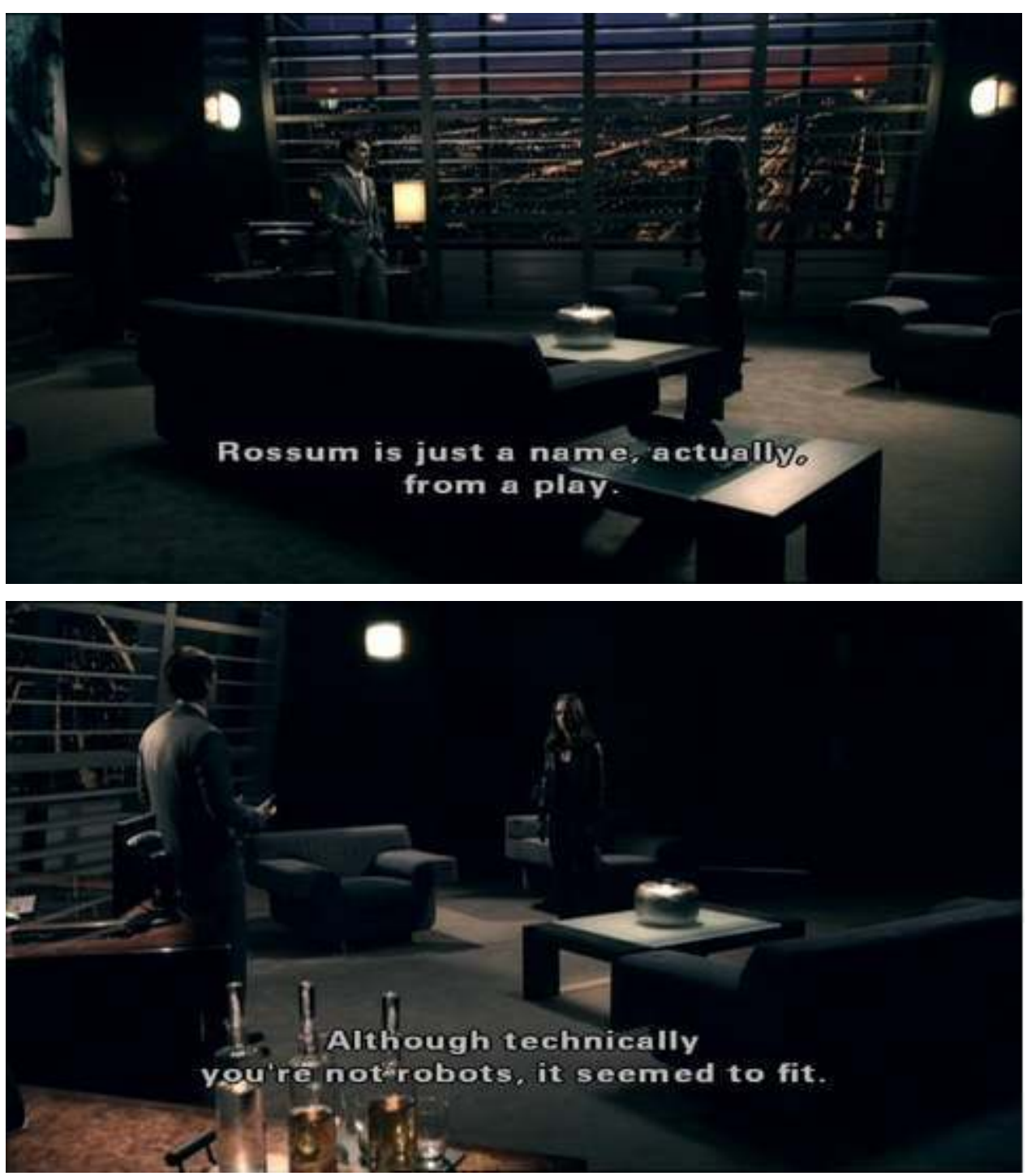

Fig. 5a, 5b : Double jeu de l'intertextualité

15 Cette utilisation dévoilée au sein de la fiction d'un nom emprunté à la pièce qui fit passer dans le langage courant le terme de « robot» (qui renvoie directement à l'esclavagisme) rend explicite le caractère aliénant de l'entreprise fictive Dollhouse tout en la renvoyant, de manière réflexive, à la littérature de science-fiction. Partageant avec l'œuvre originelle une réflexion face aux implications éthiques de la technologie, Dollhouse réactualise le propos mais y ajoute une dimension encore plus universelle.

16 En effet, le titre de la série, Dollhouse, renvoie à une autre pièce européenne, celle d'Henrik Ibsen, Une Maison de poupée (1879). Les deux œuvres mettent en scène une femme (Nora chez Ibsen, Écho chez Whedon) qui prend conscience de son asservissement et s'en libère à travers une affirmation de son individualité. La Nora d'Ibsen ne souhaite plus être définie en tant qu'épouse ou mère, - autrement dit, enfermée dans le fantasme, essentiellement masculin, des autres -, mais cherche à exister pour elle-même en étant simplement femme ${ }^{9}$. Comme l'indique Florence Fix :

Une maison de poupée propose une réflexion sur l'asservissement consenti aux autres - par habitude, lassitude ou crainte - , sur la contrainte et la servitude sociale - notamment au regard d'autrui et à la rumeur; elle soulève des 
problèmes, ouvre des questions, mais n'impose pas de solutions ou de réponses ${ }^{10}$. arences, un personnage vide, "une ardoise vierge " (" a clean slate») comme évoqué à plusieurs reprises dans la série et dès la première séquence du pilote. D’ailleurs, dès le départ, Caroline, anticipant sa singularité génétique qui la rend partiellement imperméable aux effacements de personnalité, affirme que l'on voit toujours ce qui était écrit auparavant lorsqu'on efface une ardoise. Elle s'affirme en tant que véritable palimpseste vivant, créature de Frankenstein intertextuelle (référence littéraire majeure qui innerve les fictions de Bétail nous l'avons vu), en ce qu'elle est composée d'échos de nombreuses références littéraires et/ou cinématographiques.

Les références à l'œuvre de Mary Shelley sont d'ailleurs nombreuses, comme le montre Devon Anderson, évoquant une réécriture du roman à l'ère posthumaine :

\begin{abstract}
Dollhouse retells Frankenstein with a posthuman protagonist as its focus. This hero, Echo, begins as a creation of the Dollhouse and of its lead programmer, Topher Brink. [...]. The posthuman themes in Dollhouse are readily apparent. [...]. The basic premise lies in the advanced technology that is utilized to construct the "perfect" person for any situation, requested by the Dollhouse's very rich and well-connected clientele [...]. These constructs are, in fact, a kind of psychological Frankenstein's creature-a consciousness made up of parts of many others ${ }^{11}$.
\end{abstract}

Les deux personnages, Écho et la créature de Frankenstein, partagent finalement une même quête d'émancipation et d'humanisation qui passent par une rébellion contre leurs Maitres, leurs Créateurs, ces inventeurs d'une technologie qui les a eux-mêmes transformés en Dieu, repoussant les limites connues entre la vie et la mort, le corps et l'esprit, autrement dit les paramètres qui nous définissent toutes et tous en tant qu'êtres humains. De plus, d'un point de vue formel, narratif, le roman de Shelley est construit sur une succession de récits enchâssés, de points de vue successifs qui réfléchissent les rouages même de la fiction. Dollhouse est également éminemment réflexif et métafilmique, au-delà des nombreux intertextes qui vont la construire. Car derrière l'entreprise Dollhouse, c'est l'industrie hollywoodienne qui se cache (ou se révèle).

\title{
Le posthumain au service de la critique d'Hollywood
}

Imagine a place in Los Angeles full of young, beautiful, vacuous people who strive to achieve physical perfection through constant exercise and massages. Every so often these people are sent out, dressed by a wardrobe department and accompanied by bodyguards, to play roles in other people's fantasy scenarios ${ }^{12}$. 
Cette description de Dollhouse (l'entreprise et la série) par Eve Bennett met en lumière la référence évidente à l'industrie du divertissement hollywoodienne et la critique latente qui parcourt toute la série de Whedon. Exemplairement, dans la fiction même, par l'intermédiaire du personnage comique de Topher Brink, dans l'épisode "Belle Chose " (S02E03), titre en français dans le texte, le parallèle entre les "poupées » de Dollhouse et les acteurs hollywoodiens est évoqué : "He'll be an empty-headed robot wandering around Hollywood ; he'll be fine ». La formule, cinglante, se passe de tout commentaire. De la même façon, lorsque le personnage de scientifique de la Dollhouse de Washington, Bennett Halverson (Summer Glau), découvre celle de Los Angeles dans l'épisode « Getting Closer » (S02E11), elle compare la manière dont les deux laboratoires traitent leurs "poupées » : "You let them roam. They roam like free-range chickens. We keep ours more like veal ${ }^{13}$ ". Si la comparaison animalière, provenant du personnage plutôt comique de la neuroscientifique, est destinée ici à faire sourire le téléspectateur, la dimension ironique permet à la fois de renvoyer au sort des individus posthumains privés de liberté (on pense également à la terminologie de Bétail selon Thierry Hoquet) mais aussi, de manière métafilmique, c'est-à-dire critique, aux acteurs hollywoodiens, notamment si l'on repense à la célèbre citation d'Alfred Hitchcock. D'ailleurs, pour prolonger le parallèle, les " poupées » signent des contrats de cinq ans, évoquant les fameux contrats de sept ans emblématiques de l'âge d'or hollywoodien, ces «cages dorés» dont se sont peu à peu émancipés les acteurs.

Cette proximité de la Dollhouse avec le milieu hollywoodien permet ainsi de nombreux autres rapprochements. Si les "poupées » sont des acteurs ou actrices, les personnages qui les entourent peuvent trouver leur place dans la hiérarchie hollywoodienne. Topher Brink, en ce qu'il choisit et/ou crée les personnalités implantées dans les poupées, ressemble à un scénariste, mais aussi à un metteur en scène. On le voit par exemple, de manière réflexive, dans une séquence de l'épisode "The Target » (S01E02), lorsqu'il fait jouer Boyd Langton (Harry Lennix), le protecteur d'Écho (cf. figures 6a, 6b, 6c, 6d).

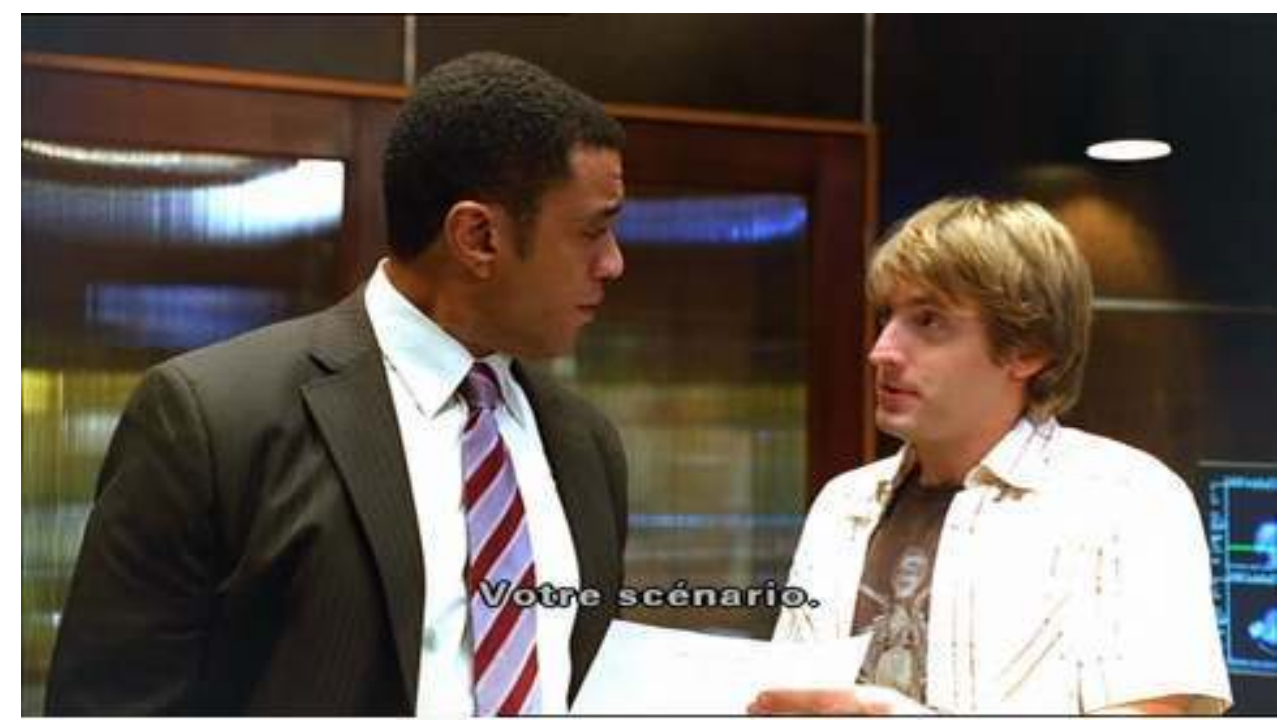



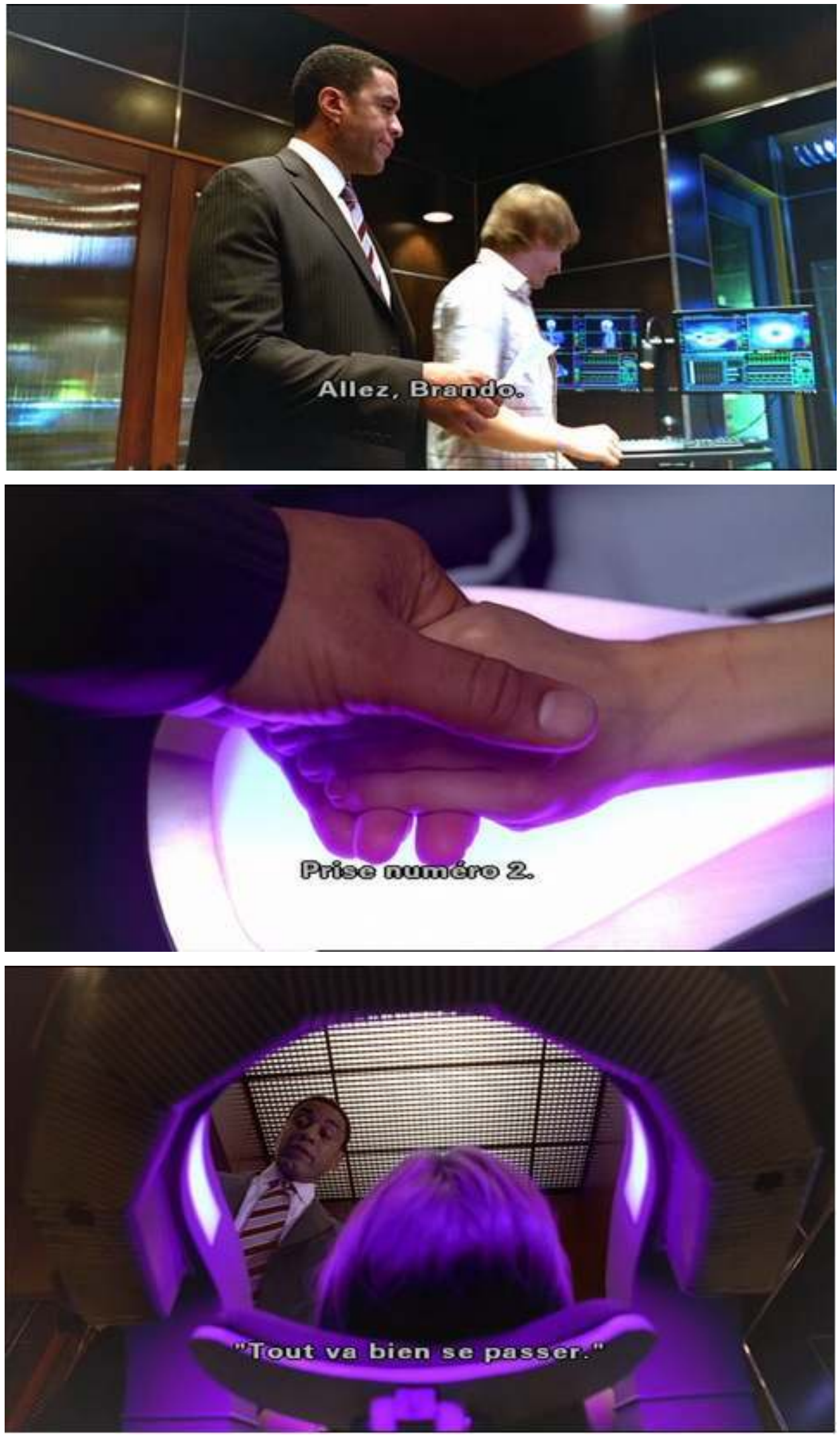

Fig. 6a, 6b, 6c, 6d : Hollywood Dollhouse

23 Les poupées de Dollhouse incarnent même parfois des personnages et/ou des acteursactrices de cinéma dans les scénarios des clients, comme par exemple la poupée Sierra 
(Dichen Lachman) prenant l'identité et l'apparence d'une sorte de Marilyn dans l'épisode «A Love Supreme » (S02E08). Ces implantations d'acteurs-actrices renvoient ainsi, par la réflexivité du procédé, les poupées à leur statut de fantasme écranique mais aussi à la performance actorale (cf. fig. 7).

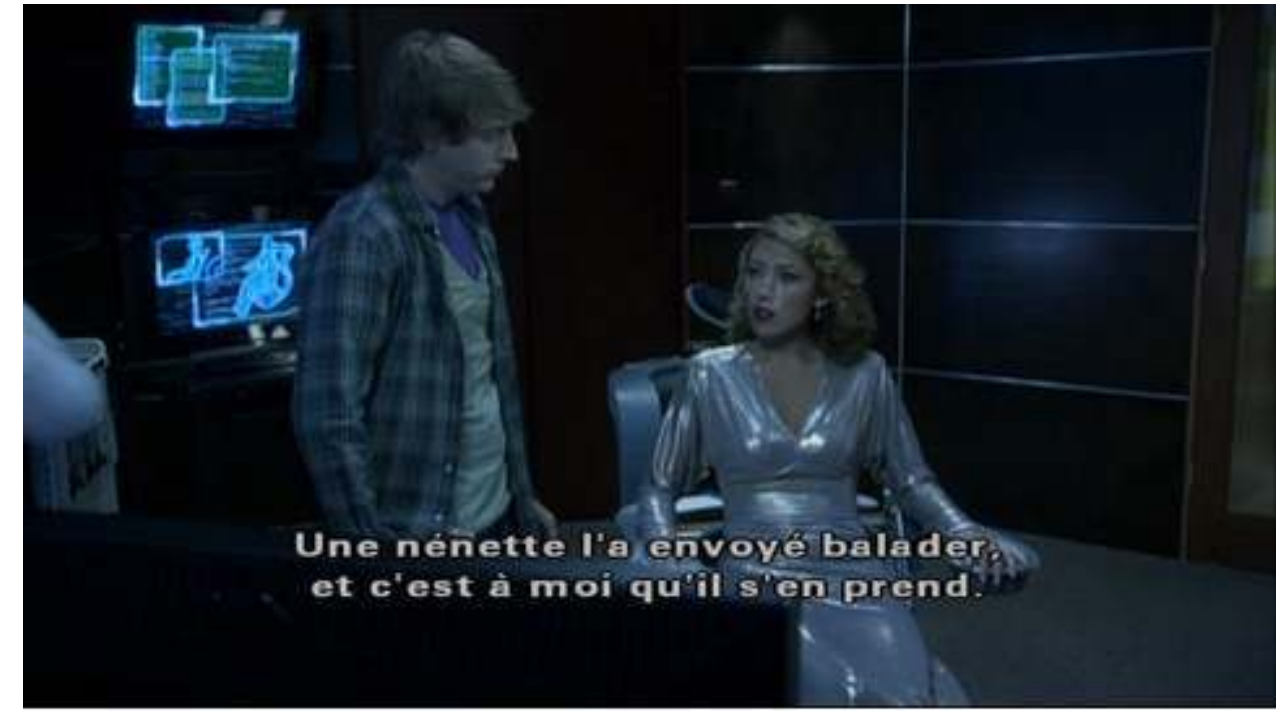

Fig. 7 : La poupée Sierra, ersatz de Marilyn?

Cette idée de performance est particulièrement mise en lumière lorsque, dans l'épisode «The Left Hand» (S02E06), la poupée Victor se voit implanter la personnalité de Topher Brink offrant à l'acteur Enver Gojkaj la possibilité de mettre en abyme (en la reproduisant) la performance d'acteur de Fran Kranz (cf. figures 8a, 8b). Il s'agit ici de rendre visible le travail des acteurs et des actrices à travers l'incarnation des personnages au sein de la fiction. Car il nous est rappelé que ce sont en réalité des acteurs qui incarnent les poupées...

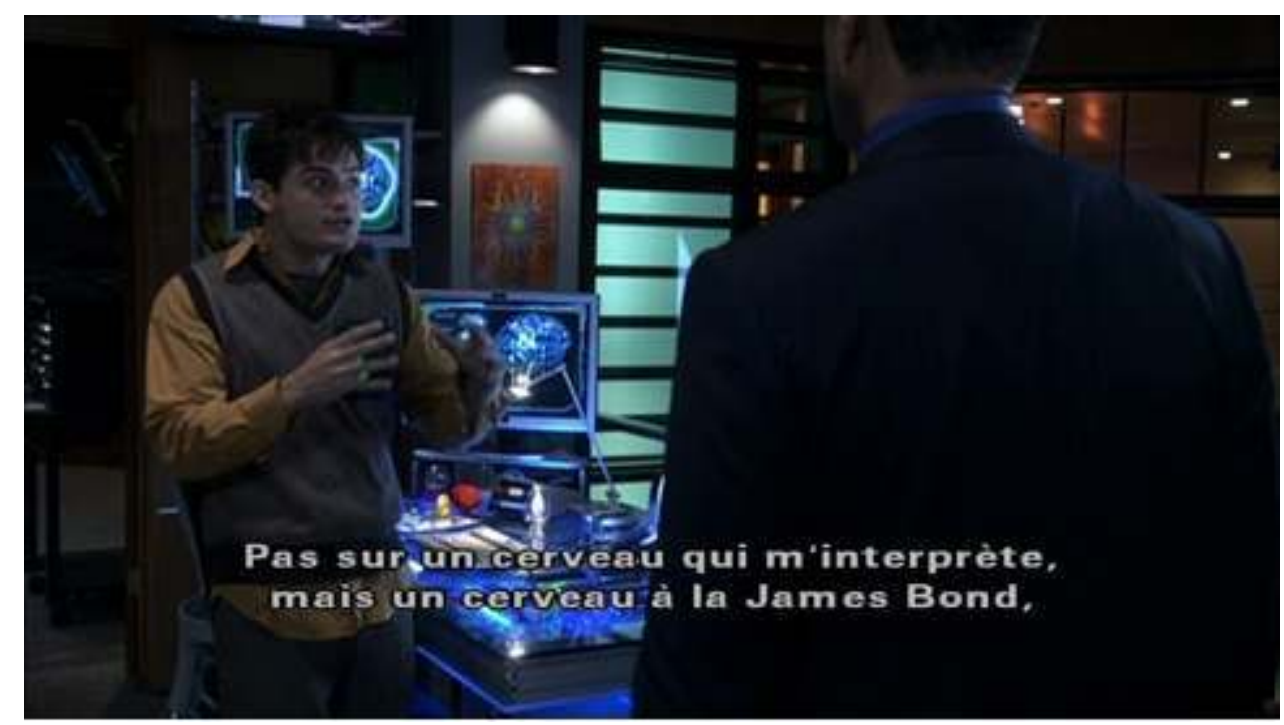




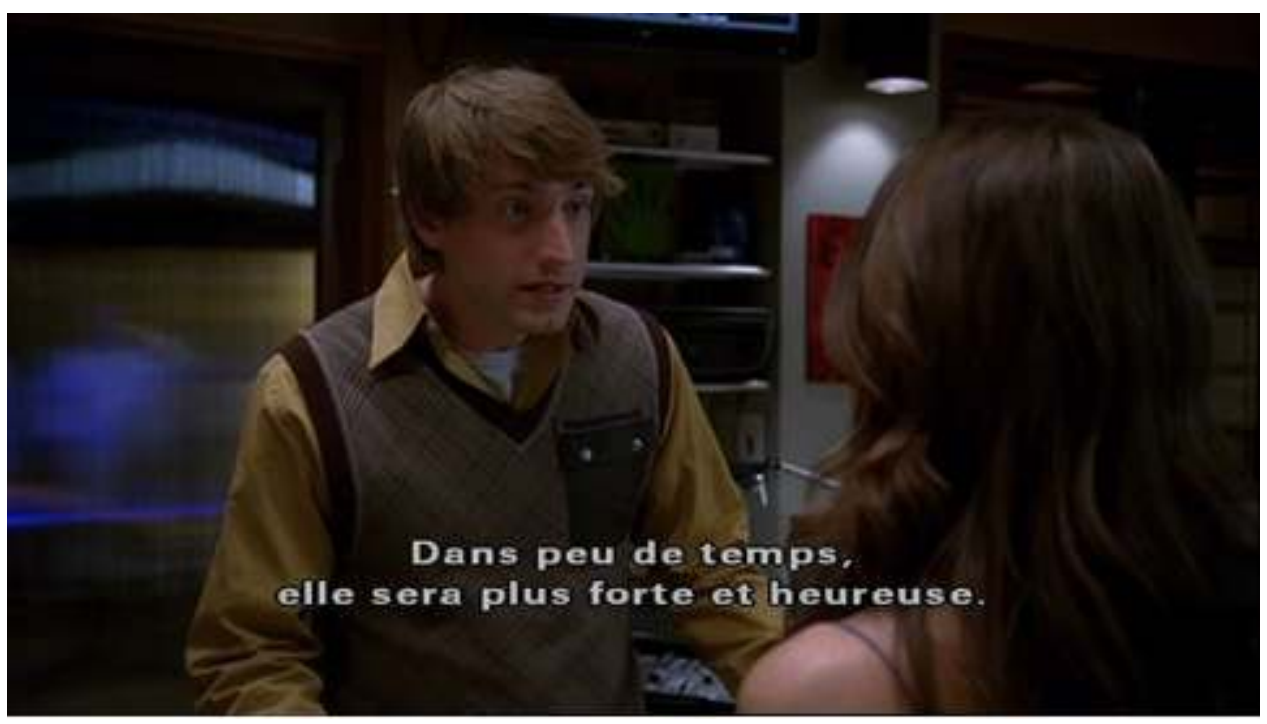

Fig. 8a, 8b : Parodier la performance actorale pour mieux la révéler (S02E06 et S01E01)

\section{Une « postsérie » au service de son actrice principale?}

Finalement on pourrait voir en Dollhouse une série qui documente le travail d'actrice d'Eliza Dushku et de ses collègues acteurs-actrices. D'autant plus que l'idée originelle de Dollhouse est née d'une discussion entre Joss Whedon et Eliza Dushku, à propos de sa carrière hollywoodienne et des rôles auxquels la jeune femme était "cantonnée ». Souhaitant offrir à son actrice une multiplicité de rôles variés pour mettre en lumière ses talents d'actrice, Whedon imagina donc le personnage posthumain d'Écho. L'origine du projet sériel est donc proprement métafilmique dans le sens où il s'agit de proposer une réflexion critique sur le travail d'Eliza Dushku au sein de l'industrie hollywoodienne. La séparation du corps et de l'esprit, thème posthumain par excellence, sert ici de prétexte pour mettre en avant les performances de l'actrice. Finalement, Eliza Dushku est semblable à la posthumaine Écho, en ce qu'elle incarne différents rôles et s'offre en fantasme au téléspectateur contre rémunération. Ainsi la série va s'efforcer d'offrir à l'actrice la plus grande variété dans ses rôles: Écho/Eliza sera ainsi une négociatrice ("Ghost », S01E01), une chanteuse («Stage Fright », S01E03), une cambrioleuse de haut vol («Gray Hour», S01E04), une aveugle faisant partie d'une secte religieuse («True Believer ", S01E05), une mère et épouse défunte («Haunted», S01E10), une travailleuse sociale ("Briar Rose », S01E11), un agent du F.B.I. infiltré ("Vows », S02E01), une jeune mère venant d'accoucher ("Instinct », S02E02) et de nombreuses petites amies idéales : au total elle incarnera environ une quarantaine de rôles au cours des 26 épisodes de la série.

Dollhouse est ainsi un "véhicule ", dans la pure tradition hollywoodienne, au service du talent d'actrice d'Eliza Dushku, comme le montre, dès les premières séquences, la référence à Cendrillon (cf. figure 9). 


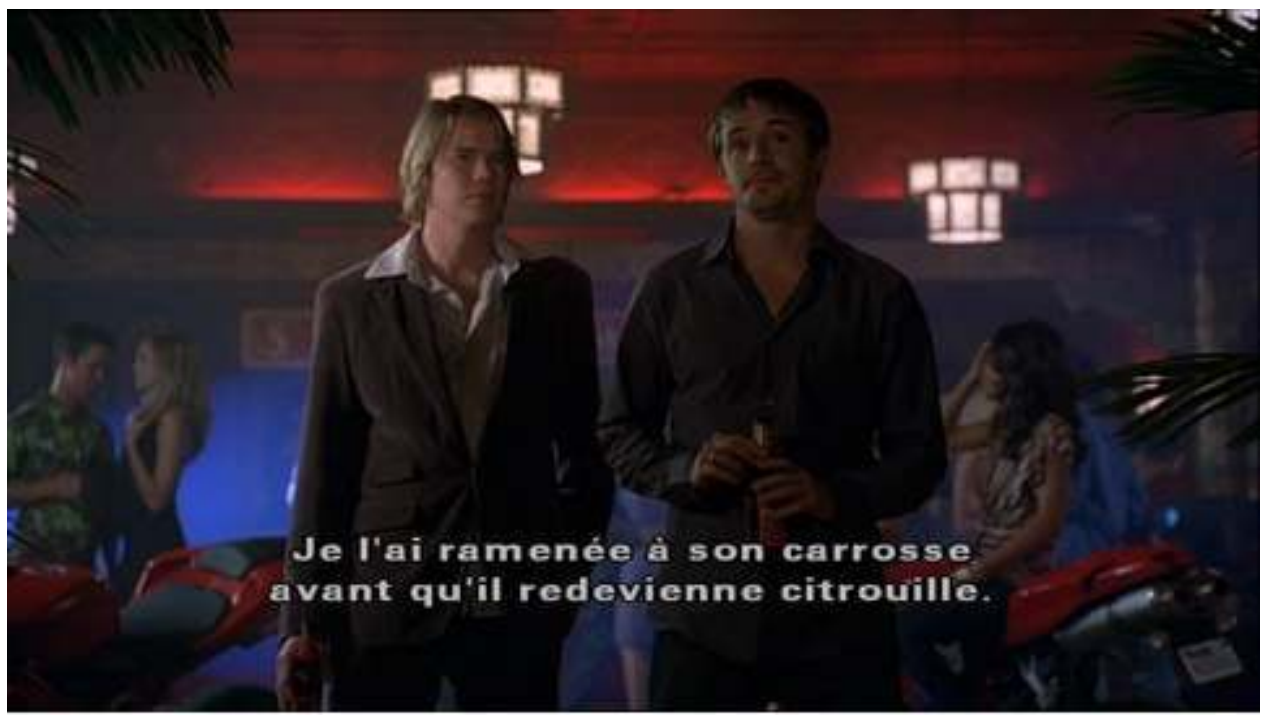

Fig. 9 : Écho, Cendrillon posthumaine?

Intertexte majeur des métafilms hollywoodiens mettant en scène l'ascension d'une star, généralement féminine, de cinéma, Cendrillon sert de canevas à la trajectoire du personnage qui, de souillon, deviendra princesse, généralement grâce à l'intermédiaire d'un producteur Pygmalion et/ou d'un Prince charmant qui aide à mettre en lumière son talent. Écho serait alors une Cendrillon posthumaine, tout à la fois souillon et princesse (son apparence dans les premières séquences du pilote la montre alternativement mal « fagotée » et en tenue de soirée - cf. figures 10a, 10b) mais aussi une multiplicité d'autres personnalités. Entre le sous-jeu nécessaire à l'interprétation du personnage d'Écho, cette coquille vide en quête d'identité, et l'exubérance qui permettra de faire exister d'autres personnages, la multiplicité des rôles interroge la capacité de l'actrice à jouer, à faire exister à l'écran les personnages devant un téléspectateur auquel on rappelle, via l'ironie permanente, que tout est finalement factice.

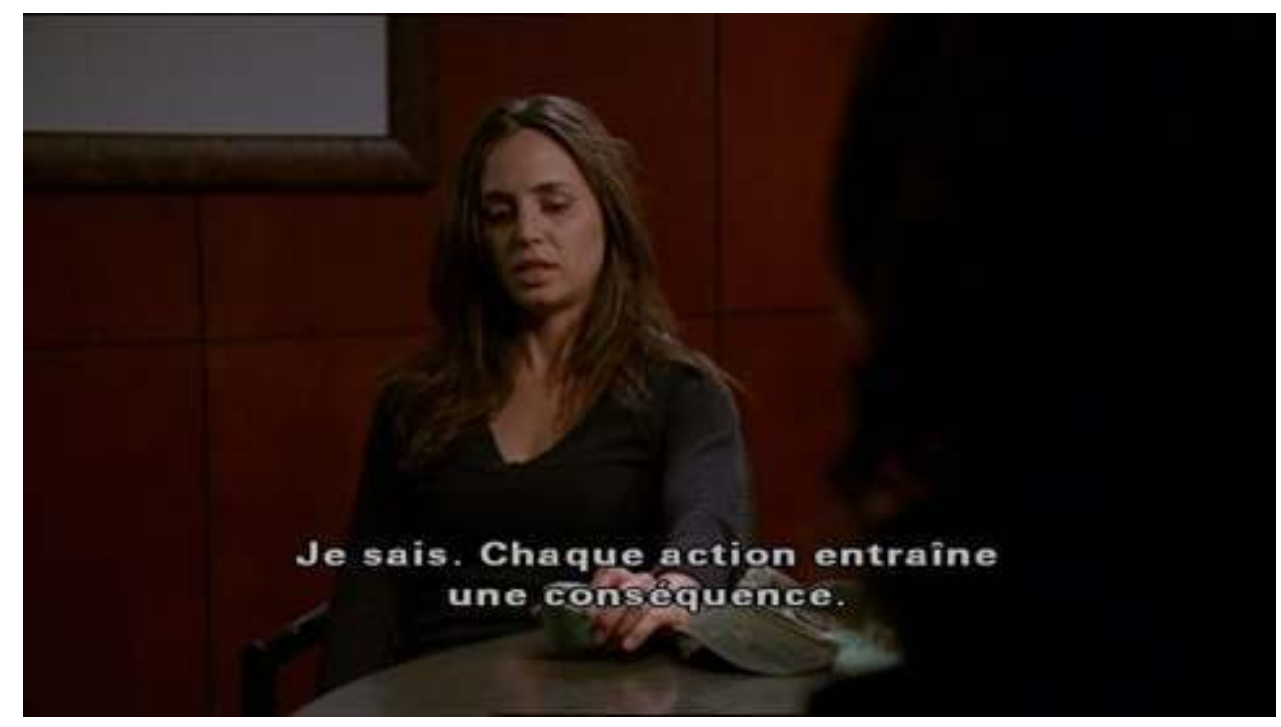




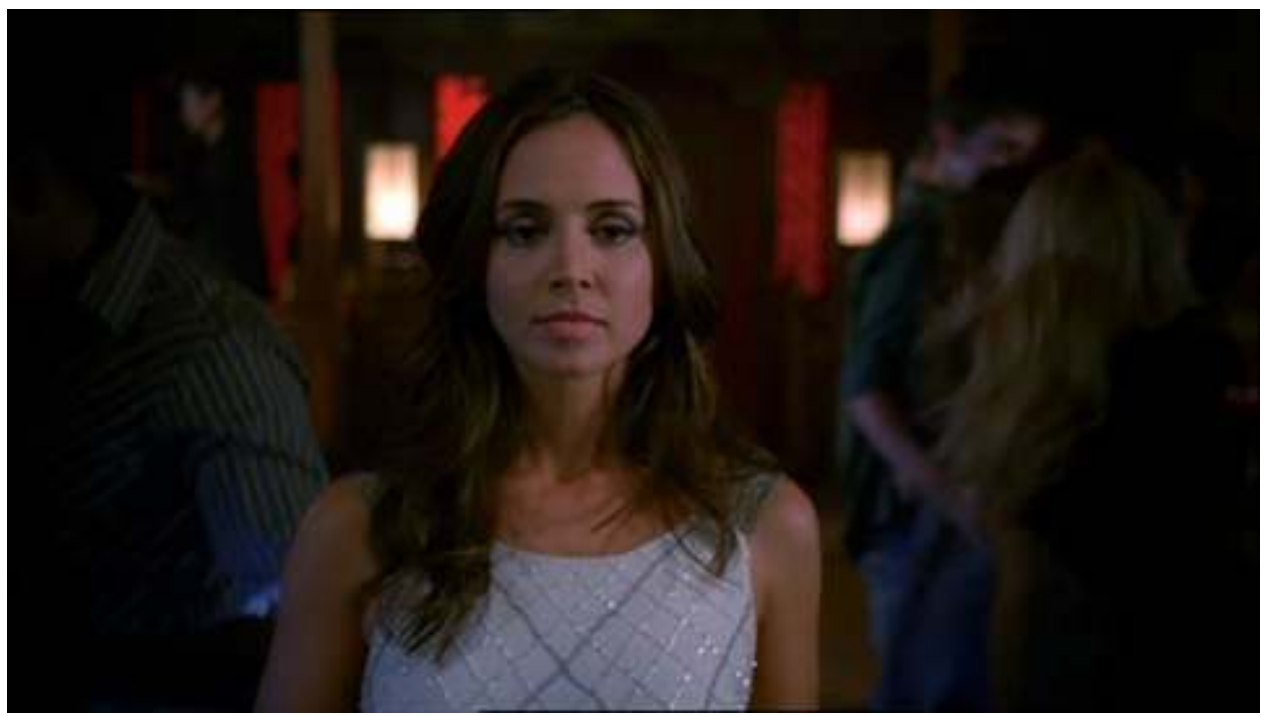

Fig. 10a, 10b : De souillon à princesse, échos de conte hollywoodien

Mais finalement Écho n'est pas une Cendrillon ou une Galatée, ni même une Belle au Bois dormant attendant d'être secourue par le Prince Charmant (cf. l'épisode «Briar Rose » qui reprend de manière subversive le conte de Grimm/Perrault - voir figure 11), elle est plutôt, comme nous l'avons vu, une créature de Frankenstein. Ce renversement permet à la série de Joss Whedon de mieux dénoncer les mythes hollywoodiens tout en offrant à sa star un rôle (ou une multiplicité de rôles) mettant en valeur son talent.

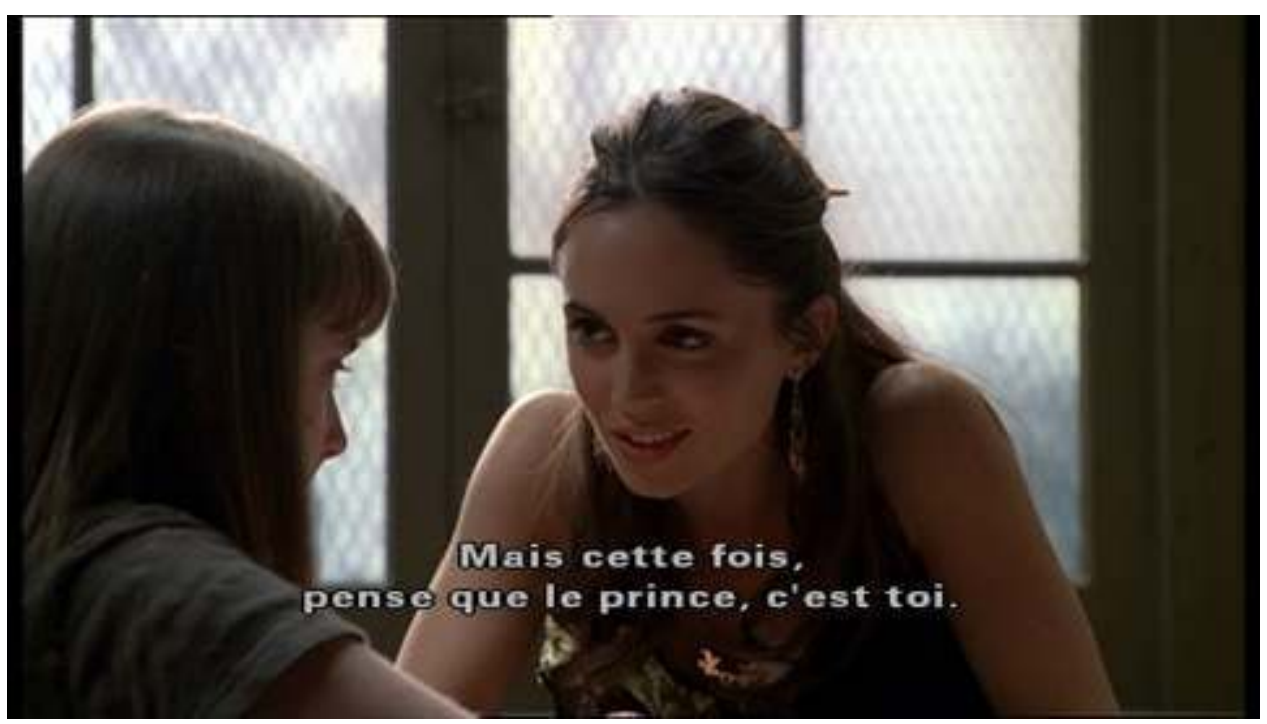

FIg. 11 : SUBVERSION DU MYTHE HOLLYWOODIEN

La technologie posthumaine offre ainsi une possibilité infinie de rôles à interpréter aux acteurs mais contamine également la structure même de la série. Dollhouse est ainsi telle une série « améliorée " par la posthumanité de son personnage principal en tant qu'elle peut, elle aussi, arpenter une potentialité infinie de genres, de registres à travers autant d'histoires, d'intrigues épisodiques, ou de (micro-)récits (et les manières de les construire). Chaque nouvelle identité, stockée sur les disques durs qui deviennent autant de réservoirs numériques (duplicables à l'infini) de fictions, est une promesse de potentielle reformulation de la série (représentée par le mur d'images qui clôt chaque épisode). 

certes pas originale et découle d'un cahier des charges imposé par le réseau de diffusion FOX ${ }^{14}$ mais elle trouve dans son personnage posthumain central l'objet idéal pour assurer, de manière explicite, métafilmique (et ironique) l'ambition de son créateur de faire œuvre. Comme Écho la posthumaine, Whedon doit répondre a une injonction paradoxale qui définit la sérialité télévisuelle: être toujours différent à travers l'épisodique (et l'intrigue de la semaine) et conquérir son identité propre à travers le feuilletonnant. Entre Alias et Code Quantum, Dollhouse propose donc un macro-récit paranoïaque d'espionnage augmenté d'un univers de science-fiction dystopique, et des micro-récits qui permettent à la série d'arpenter différents genres dans les épisodes : thriller, policier, fantastique («Haunted», S01E10), film de braquage (« Gray Hour », S01E4), survival (« The Target », S01E02), espionnage ( A Spy in the House of Love», S01E09), mélodrame (« Instinct », S02E02), film de tueur en série (« Belle Chose », S02E03), etc. Mais également de varier les registres et les genres au sein des épisodes, passant du thriller à la comédie. Les épisodes eux-mêmes, dans la structuration du récit, proposent des reformulations permanentes: l'épisode "The Target» (S01E02) est construit grâce à de multiples analepses, tandis que l'épisode « A Spy in the House of Love » (S01E09) utilise les points de vue de quatre personnages différents pour construire son récit.

l'avons dit, Dollhouse est une série de science-fiction ; mais elle ne se déroule pas dans le futur, préférant s'inscrire dans son époque contemporaine immédiate (2009-2010). Toutefois les deux derniers épisodes (des deux saisons) se déroulent dix ans après le récit initial, en 2019 et 2020, et dévoilent un monde post-apocalyptique et cyber-punk rappelant tout à la fois Mad Max (George Miller, 1979) et Matrix (Andy et Larry Wachowski, 1999), ce dernier figurant parmi les films préférés de Joss Whedon. Ce saut dans le futur $\mathrm{du}$ récit, reformulant encore la série, met en lumière plus directement le genre de la dystopie auquel elle se rattache. Dollhouse nous propose ainsi une réalité alternative et imagine le danger d'utiliser d'une technologie qui pourrait aboutir à l'extinction de l'humanité. Comme le rappelle Thierry Hoquet :

Le but de la SF est d'explorer des potentialités latentes dans la réalité, et de réunir ainsi la dimension fictionnelle (non mimétique) tout en nous faisant voir ce qui se trouve enfoui dans la réalité concrète. C'est une logique de l'extrapolation. La science-fiction mobilise un regard critique sur le monde: elle ouvre des possibles si bien que plus rien ne sera jamais comme avant ${ }^{15}$.

L'utilisation de la SF dans Dollhouse fonctionne ainsi à de multiples niveaux, grâce à sa dimension réflexive.

Dollhouse s'appuie sur un univers diégétique posthumain et dystopique pour dénoncer l'aliénation à l'œuvre dans notre société contemporaine. De manière réflexive, c'est aussi une critique de l'industrie audiovisuelle hollywoodienne où les acteurs et actrices sont comparés à des poupées ou à du Bétail. Mais cette dénonciation, qui émane du cœur du système que la série dénonce, permet aussi de mettre en lumière de manière frappante le talent d'actrice Eliza Dushku, dans la tradition du «véhicule» hollywoodien, en lui offrant la possibilité de s'émanciper des rôles auxquels elle était cantonnée, justement, à cause de son image de "poupée ». La posthumanité à l'œuvre contamine la structure même de la série et permet à son auteur d'amener les spectateurs à réfléchir à l'assemblage de l'épisodique et du feuilletonnant qui constitue l'essence de l'œuvre 
Dollhouse, mise en lumière dans sa mise en abyme de l'articulation entre posthumanité et série.

\section{NOTES}

1. J.J. Abrams, ABC, 2001-2006.

2. Donald P. Bellisario, NBC, 1989-1993.

3. Dominique Lecourt, Humain Posthumain. La technique et la vie, Paris, Presses Universitaires de France, collection «Histoire et société ", avril 2003, p. 70 : "La scène primitive a sans doute été inventée par Hans Moravec dans Mind Children: The Future of Robot and Human Intelligence [en 1988]. Elle montre un être humain téléchargeant son esprit sur un ordinateur par une manière de liposuccion crânienne. »

4. Ibid., p. 120.

5. François-Ronan Dubois, «Les traits définitoires de l'humain dans la série Doctor Who» in PostHumains. Frontières, évolutions, hybridités, éd. Elaine Després, Hélène Machinal, Rennes, Presses Universitaires de Rennes, collection «Interférences », 2014, p. 181.

6. D'ailleurs, l'explication scientifique de cette altération n'est pas développée et/ou véritablement montrée dans la série. Le cerveau est relié sommairement à des électrodes lors du processus.

7. Thierry Hoquet, «Cyborg, Mutant, Robot, etc. Essai de typologie des presque-humains » in PostHumains. Frontières, évolutions, hybridités, op. cit., p. 100-101.

8. Ophélie Hernandez, « Polymorphie du corps post-humain », Le post-humain et les enjeux du sujet, éd. Xavier Lambert, Paris, L'Harmattan, 2011, p. 53-54.

9. «Ici, j'ai été ton épouse-poupée, tout comme à la maison, j'étais l'enfant-poupée de papa. Et mes enfants, à leur tour, ont été mes poupées. Je trouvais divertissant que tu te mettes à jouer avec moi, tout comme ils trouvent divertissant que je me mette à jouer avec eux. Voilà ce qu'a été notre mariage, Torvald. » Et aussi : "Je crois que je suis d'abord et avant tout un être humain, au même titre que toi... ou, en tout cas, que je dois essayer de le devenir. [...]. Il faut que je réfléchisse moi-même à ces choses et tâche de voir clair en elles. » Henrik Ibsen, Une maison de poupée, Acte III, 1879, traduction Régis Boyer, Éditions Flammarion, 2016, p. 214 et 218, nous soulignons.

10. Florence Fix, « Dossier » in Henrik Ibsen, Une maison de poupée, op.cit., p. 228-229.

11. Devon Anderson, «Echoes of Frankenstein: Shelley's Masterpiece in Joss Whedon's Dollhouse and Our Relationship with Technology » in Slayage: The Journal of Whedon Studies, 14.1 [43], Hiver 2016, disponible à l'adresse suivante: http://www.whedonstudies.tv/ uploads/2/6/2/8/26288593/anderson_slayage_14.1.pdf.

12. Eve Bennett, «Deconstructing the Dream Factory: Personal Fantasy and Corporate Manipulation in Joss Whedon's Dollhouse » in Slayage: The Journal of Whedon Studies, 14.1 [43], Hiver 2016, disponible à l'adresse suivante :

http://www.whedonstudies.tv/uploads/2/6/2/8/26288593/bennett_slayage_9.1.pdf. 
13. «Vous les laissez en liberté, courir comme des poulets en plein air. On parque les nôtres comme des veaux. »

14. Joss Whedon, « Dollhouse: Joss Whedon Q\&A» in http:// sepinwall.blogspot.fr/2009/02/dollhouse-joss-whedon-q.html, consulté le 4 janvier 2018 : "The mandate from the network was very much, "Give me a stand-alone where the audience knows what the structure of the show is, and where it ends so the audience wants to come back next week, but not just because you put out a carrot and they know they're going to be chasing that carrot for six years." And I agree with that. Some of the best stuff we're doing with the show is arc stuff, but at the same time, if the adventure of the week isn't compelling, and it's just a string of compelling scenes where nothing is resolved - that kind of television frustrates me enormously. I'd say we want to do both. We want people to feel like they can watch it when they want to, week to week, or they can watch a 13-hour movie. »

15. Hoquet, op. cit., p. 103.

\section{RÉSUMÉS}

Dollhouse de Joss Whedon est une série mettant en scène Écho, un personnage posthumain asservi, ayant la faculté d'acquérir un nombre illimité de personnalités afin d'accomplir différentes missions. En quête d'émancipation, Écho va lutter au sein d'un système qui, de par la réflexivité et l'intertextualité déployées dans la série, possède de nombreux points communs avec l'industrie hollywoodienne du divertissement. Série hybride, Dollhouse sert de « véhicule » à son interprète principale, Eliza Dushku, tout en réfléchissant sur l'articulation entre l'épisodique et le feuilletonnant.

Joss Whedon's series Dollhouse features Echo, an enslaved posthuman character with the ability to acquire an unlimited number of personalities to accomplish different missions. In search of emancipation, Echo will fight in a system that, through the reflexivity and intertextuality deployed in the series, has many points in common with the Hollywood film industry. A hybrid series, Dollhouse serves as a "vehicle" for its main star Eliza Dushku, while reflecting on the articulation between episodic and serial.

\section{INDEX}

Mots-clés : Dollhouse, Dushku Eliza, épisodique, feuilletonnant, Hollywood, intertextualité, posthumain, réflexivité, Whedon Joss

Keywords : Dollhouse, Dushku Eliza, episodic, Hollywood, intertextuality, posthuman, reflexivity, serial, Whedon Joss 


\section{AUTEUR}

\section{JULIEN ACHEMCHAME}

Julien Achemchame est enseignant certifié de lettres modernes-cinéma, docteur en études cinématographiques et chargé d'enseignement à l'Université Paul-Valéry Montpellier 3. Auteur de deux ouvrages sur le cinéaste américain David Lynch, ses recherches actuelles s'orientent principalement vers la circulation des formes et des motifs filmiques dans l'histoire du cinéma, notamment à travers l'exemple des films réflexifs et, en particulier, du métafilm hollywoodien, et aussi vers l'analyse des formes de sérialité à l'œuvre dans les séries télévisées contemporaines. Julien Achemchame holds a PhD in film studies. He teaches film in high school and at Paul-Valéry University in Montpellier (France). The author of two books on the American filmmaker David Lynch, his current research focuses mainly on the circulation of filmic forms in Cinema history, especially through the example of reflexive films and, in particular, Hollywood metafilms, and on the analysis of the forms of seriality in contemporary television series. 